\title{
The Structure of Cellulose and Related Substances
}

\section{1} $\mathrm{HE}$ discussion arranged by Section B (Chemistry) of the British Association on September 2, on the constitution of polysaccharides with special reference to fibres, was perhaps a happy example of what such discussions should be and disarms some of the criticism which has recently been levelled against the programmes of certain of the sections. The results of recent researches in this difficult but fascinating field were presented for the most part in a lucid manner which rendered them intelligible to scientific workers generally and not merely to specialists in this particular field. For this some credit must undoubtedly be given to the formal arrangement of the discussion; the gap left by Prof. M. Bergman, who was unable to be present, was well filled by Prof. L. Zechmeister.

Prof. W. N. Haworth opened the discussion with a survey of the development of our knowledge of the constitution of polysaccharides in which the fundamental contribution of the work of the Birmingham school in establishing the structure of the mono- and di-saccharides was emphasised. The structure of cellulose, for example, rests ultimately on the constitution assigned to the disaccharide cellobiose, and the mutual linking of $\beta$-glucopyranose residues in a chain through positions 1 and 4 of the glucose molecules is the fundamental principle of the modern cellulose structure. Recent work by Haworth and Machemer has indicated that information on the approximate length of the chains and as to whether they are open or closed can be obtained by a study of the fully methylated derivative of cellulose, obtained by methylation under very mild conditions in which no important degradation or decomposition of the macro-molecules occurs. Since if the macromolecule is in the form of an endless chain, each glucose unit ultimately yields $2: 3: 6$-trimethylglucose, while if the chain terminates one of the terminal groups yields a molecule of tetramethylglucose, and a quantitative separation of the tri- and tetramethylglucose is possible, the hydrolysis method can be used to determine the structure of the chain. The formation of $\mathbf{0 . 6}$ per cent of tetramethylglucose accordingly indicates a mean average length of about 100-200 glucose units for the terminated chains in cellulose. The average molecular weight of 30,000 thus indicated is in good agreement with values obtained from X-ray data and by the use of Svedberg's ultra-centrifuge method. Values obtained by this method for the chain length of a long series of cellodextrins are in excellent agreement with those obtained by viscosity measurements or by determination of the iodine value.

Prof. H. Staudinger then described the viscosity method of investigating the nature and size of the colloid particles of cellulose and related substances. An exhaustive study of synthetic polymeric sub. stances of known constitution parallel with that of the 'polymer homologous' series of degradation products of cellulose showed that in sufficiently dilute solution the relation between their molecular length and viscosity in solution is expressed by the viscosity law :

$$
\eta / c=K_{m} \cdot M
$$

where $\eta$ is the specific viscosity, $c$, the concentration, $K_{m}$ a characteristic constant for each polymer homologous series and $M$ the molecular weight. In these solutions as in solutions of cellulose in Schweizer's reagent or of cellulose acetate or nitrate in organic media the colloid particles are the molecules them. selves and do not have a micellar structure. The 'polymer homologous' series of degradation products of cellulose obey the same law and these methods lead by extrapolation to a molecular weight of 120,000 for cellulose in Schweizer's reagent, 750 glucose residues being combined in one molecule. The mole. cules are long threads which in one dimension are 500 times longer than in the other two. Viscosity relations also afford evidence of the presence of a 6-carbon atom ring in the glucose residues of cellulose.

Prof. L. Zechmeister's paper discussed the unex. pected behaviour towards enzymes of polysaccharides of comparatively small chain-length (4-8 glucose units). Dr. Zechmeister suggested that in enzyme reactions the length of chain of the reacting molecule as well as the character of the groupings present is important. The field of investigation thus outlined is of the greater interest in view of Mr. W. T. Astbury's suggestion of the existence of a fundamental relation between protein chains and polysaccharide chains.

Dr. E. L. Hirst then discussed the question of the molecular structure of starch and its allied products glycogen and inulin. In starch the mutually linked glucose residues are united by $\alpha$-glucosidic linkings in contrast with the $\beta$-glucosidic linkings of cellulose, while the $\alpha$-linkings of starch do not readily give a straight chain pattern but favour the formation of interlocked aggregates of the macro-molecules, which in presence of water are hydrated with formation of micellar solutions. Recent work has shown that the differences between amylose and amylopectin do not depend on the phosphorus content. On careful acetylation and methylation the special characteristics of each modification are retained and since the purified and fractionated methylated derivatives both yielded 5 per cent of tetramethylglucose, the macromolecules of amylose and amylopectin both consist of terminated chains of 24-30 glucose units (mean average mol. wt. 4000-5000) and the differences between amylose and amylopectin depend on hydra. tion and interlocking of the macro-molecules and on micellar structure. Glycogen is built up on a plan similar to that of starch and differs from amylose in having a macro-molecule containing only 12-14 glucose residues in the chain. Similarly inulin has been found to consist of a terminated chain of about 30 fructofuranose residues mutually linked through positions 1 and 2 , the macro-molecule probably terminating at one end in a reducing group. The corresponding molecular weight (about 5000) is in good agreement with estimates made by direct methods.

Prof. H. Mark gave a critical review of the bases upon which the accepted formula for cellulose rests, and showed how a combination of the chemical methods which had established the structure of cellobiose and the disaccharides, the application of $\mathrm{X}$-ray methods, and the researches of Prof. Staudinger on the nature and size of molecules of colloidal substances had led to a picture of the cellulose molecule and of the arrangement of molecules in the fibre which in many respects may be considered as defi. nitely established. From a detailed examination of $\mathrm{X}$-ray data including data recently obtained by the

No. 3287, Vol. 130] 
application of new methods Dr. Mark described the arrangement of the glucose units in the fibre and of groups of these chains associated as micellar bundles, the length of the chain being about 100-200 glucose residues, in agreement with the value obtained by the chernical methods of the Birmingham school.

The final paper by Mr. W. T. Astbury described the application of X-ray methods in the field of protein chemistry, where again the combination of the results of organic chemistry and physical methods has established the concept of long chain molecules. Protein fibres are built up of polypeptide chains in various states of extension whereas cellulose appears to be laid down in biological structures as fully extended chains. X-ray methods have as yet revealed only two proteins, the fibroin of natural silk and the $\beta$-keratin of stretched hair which are in a fully extended state. Since in natural processes the formation of cellulose and other polysaccharides seems to be effected through the intervention of proteins, it is possible that the protein chains may act as a pattern or framework upon which the sugar units are laid down as a preliminary step to their linking together in polysaccharide chains. It is an interesting point that the chief longitudinal spacings of muscle and of unstretched hair are almost equal to the length of a glucose residue as it occurs in cellulose and the fact that the crossed-cellulose chains of the wall of Valonia ventricosa are laid down according to a definite plan indicates that they have been built on a net-work pattern in the underlying protoplasmic layer.

\section{Sixth International Congress of Genetics}

OTWITHSTANDING the small number of
European delegates attending, the International Congress of Genetics held at Cornell University, Ithaca, New York, on August 24-31, was a marked success. This was mainly owing to the large amount of preparatory work undertaken by the Organisation Committee, the Executive Council and the Local Committee. Although many papers were not read in the absence of their authors, the programme was still overcrowded, and the volume of proceedings containing the abstracts of papers and descriptions of exhibits runs to more than four hundred pages. The exhibits alone would have furnished ample material for a busy week. Numerous laboratories in several buildings were devoted to exhibits, in which cytological demonstrations played a prominent part; but they included also living and dried specimens illustrating genetic experiments with fungi, liverworts, mosses, ferns, numerous cereals, economic and other plants, as well as collections of varieties of maize and vegetables and numerous floricultural exhibits. The animal exhibits included Drosophilia and Sciara, Lepidoptera, Orthoptera, bees, aphids, Gammarus, tunicates, echinoderms, molluses, fishes, rats and mice, foxes, pigeons, guinea-pigs, and all the domestic animals, many of the latter as living specimens.

A unique feature of the Congress was the genetic garden (Fig. 1), contributed to by geneticists who had sent seeds from many parts of the world. Here were demonstrated and compared side by side the various forms, hybrids and mutations, of Zea, Enothera, Nicotiana, Primula, Antirrhinum, Pharbitis, Helianthus, Pisum, etc. A special feature arranged by Prof. Emerson was the display of the numerous mutations of Zea in their proper order in the ten chromosomes, as determined by linkage investigations. A chart also showed the loci of more than eighty genes in maize.

The cytological demonstrations were so many that, although a hundred microscopes must have been in use, each exhibit could only be set up for one afternoon. The work on maize chromosomes by the Cornell group was the basis of excited discussions. The great detail of ehromosome structure and behaviour observable in maize is in marked contrast to Drosophila, although here, too, numerous cases of visible translocations were demonstrated.

The morning sessions were devoted to general papers grouped under such topics as the nature and cause of mutations, the interrelations of cytology

$$
\text { No. 3287, Vol. 130] }
$$

